\title{
Submicron metal oxide structures by a sol-gel process on patterned substrates
}

\author{
Clemens Bechinger*, Hans Muffler, Claudia Schäfle, Olle Sundberg, Paul Leiderer \\ University of Konstanz, Fach $M 676$, D-78457 Konstanz, Gemany
}

Accepted 27 January 2000

\begin{abstract}
We report about a combination of micro-contact-printing and the sol-gel technique which results in structures in the micron- and submicron range. This technique which is here demonstrated on electrochromic tungsten oxide does not rely on vacuum methods and may therefore be easily upscaled to large areas. (C 2000 Elsevier Science S.A. All rights reserved.
\end{abstract}

Keywords: Nanostructures; Titanium oxide; Tungsten oxide; Wetting

\section{Introduction}

The sol-gel technique is a widespread method of synthesizing materials and depositing them in form of thin homogeneous films. Accordingly, it has gained importance with respect to scientific and application purposes. Based on the chemistry of the synthesis, a large variety of materials, e.g. oxidic semiconductors, glasses and even superconducting films can be obtained [1-4]. In this process an inorganic or organic molecular precursor $\mathrm{M}(\mathrm{OR})_{n}$, a sol, is used as starting material, where $\mathrm{M}$ is a metal and (OR) an organic compound, respectively. A macromolecular oxide network is then obtained in a moist environment through hydrolysis and polycondensation which transforms the precursor into a highly viscous (gel-like) hydrated metal oxide $\left(\mathrm{MO}_{x}\right)$. In a final heat treatment at typically $100-300^{\circ} \mathrm{C}$ the coating is then dried in an oven to complete hydrolysis and condensation. In principle the sol-gel reaction can be written as [1]

$\mathrm{M}(\mathrm{OR})_{n}+n / 2 \mathrm{H}_{2} \mathrm{O} \rightarrow \mathrm{MO}_{n / 2}+n \mathrm{ROH}$

The basic advantage of the sol-gel process is its ability to form inorganic structures (semiconductors, ceramics and glasses) at relatively low temperatures. Furthermore, the process - being very similar to conventional coating techniques such as dip-, spin- or spray coating, does not require any vacuum steps, and is therefore a cost-effective way to produce thin homogeneous inorganic films on large scales.

In the following we will demonstrate that this technique -

* Corresponding author. Tel: + 49-75-31-883-562; fax: + 49-753188-3127.

E-mail address: clemens.bechinger@uni-konstanz.de (C. Bechinger) in contrast to homogeneous thin film deposition as it has been used so far - can also be employed for the fabrication of nanostructures on large scales which are useful for electronic, optical or catalytic applications. This is achieved by combining the benefit of the sol-gel process with microcontact-printing $(\mu-\mathrm{CP})$, the latter comprising surfaces with well-defined periodic hydrophilic and hydrophobic domains, respectively $[5,6]$. When a medium of low viscosity is applied to such patterned surfaces, spontaneous dewetting occurs and the fluid forms a droplet pattern which reflects the symmetry of the underlying substrate. The principle has been demonstrated already for simple liquids, polymers and other materials $[7-10]$ on homogeneous substrates. Additionally, fabrication of patterned sol-gel structures by means of micromolding is reported in the literature $[11,12]$. In contrast to this, here we apply highly reactive liquids, i.e. alkoxides, to chemically patterned substrates. After the dewetting process an array of drops is obtained which can be considered as microscopic test tubes where a chemical reaction, namely the sol-gel process takes place, which eventually turns the liquid drops in solid dots.

\section{Sample preparation}

We demonstrate the principle for the semiconductor tungsten oxide $\left(\mathrm{WO}_{3}\right)$ which can be prepared from a tungsten alkoxide (or alcoholate) precursor, but the principle should hold for many other oxidic materials. We choose $\mathrm{WO}_{3}$ since it is well known as a chromogenic material which can change its optical and electrical properties $[13,14]$ upon 


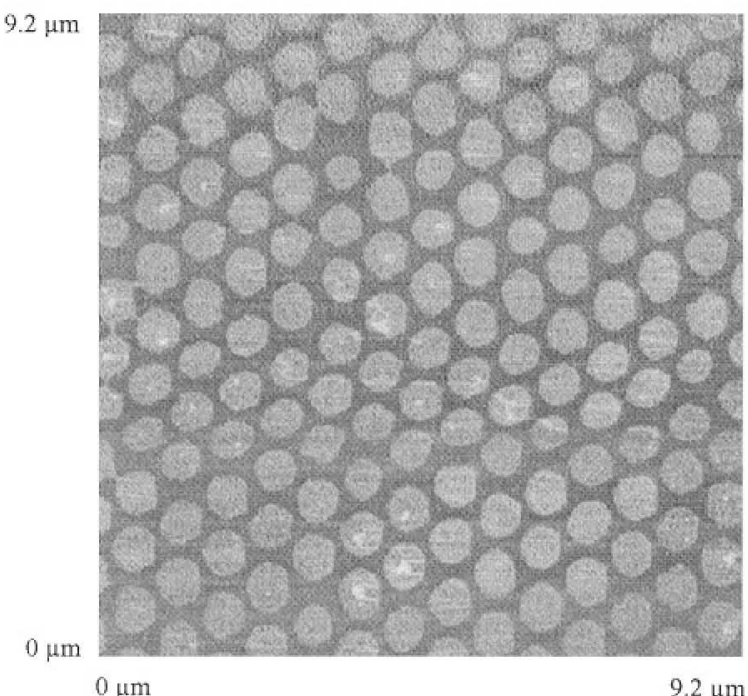

Fig. 1. Lateral force image of a chemically patterned gold surface where bright and dark areas indicate hydrophilic and hydrophobic domains, respectively. The structure was obtained by micro-contact printing using a monolayer of polystyrene spheres of $840 \mathrm{~nm}$ diameter as a master.

applying electrical fields, irradiation with ultraviolet light [15] or exposure to hydrogen containing gases [16]. Therefore the material is interesting for potential applications in displays, imaging devices and "smart" windows $[17,18]$. Since the presented approach does not require vacuum or etching techniques it might be a promising method for the fabrication of oxidic nanostructures on large scales.

Tungsten alkoxide precursors were prepared by dissolving $3 \mathrm{~g}$ tungsten hexachloride $\left(\mathrm{WCl}_{6}\right)$ in $10 \mathrm{ml}$ ethanol. First a violent reaction occurs which turns the yellow solution rapidly into blue. To avoid the gelation to take place already during the deposition process of the sol, we stabilized it against hydrolysis by adding $10-20 \mathrm{ml}$ acetylacetone as a surfactant $\left(\mathrm{C}_{5} \mathrm{H}_{8} \mathrm{O}_{2}\right)$ [19]. The final mixture contained also $7 \mathrm{ml}$ dilute $(1 \mathrm{M})$ hydrochloric acid in order to enhance the dewetting properties. When this liquid is applied by dip or spin coating to a homogeneous substrate and annealed at about $120^{\circ} \mathrm{C}$, a thin film of tungsten oxide $\left(\mathrm{WO}_{3}\right)$ is formed which exhibits a highly reversible electrochromic behavior comparable to that of evaporated or sputtered material $[3,20,21]$.

Chemically patterned substrates were fabricated with self-assembled monolayers (SAMs) on gold coated glass sheets by using combinations of octadecanethiol $\left(\mathrm{CH}_{3}\left(\mathrm{CH}_{2}\right)_{17} \mathrm{SH}\right)$ and 11-mercaptoundecanol $\left(\mathrm{HS}\left(\mathrm{CH}_{2}\right)_{11} \mathrm{OH}\right)$, resulting in hydrophobic and hydrophilic domains, respectively. To transfer the thiols to the surface a stamp of an elastomeric material is required. Stamps can be obtained by casting poly(dimethylsiloxane) (PDMS) onto an appropriate master. A negative image of the master is produced in the cured PDMS. Conventional methods as photolithography or X-ray lithography can be used to produce the masters. Here, however, we used a different technique which has become known as nanosphere lithography, and which works in principle as follows [22-24]: colloidal particles of equal size which are suspended in a solvent (e.g. water) are deposited on a glass surface. The particles usually arrange themselves randomly. However, during the evaporation of the solvent, lateral capillary forces occur which make the colloids to form hexagonally arranged, close-packed arrays in a self-assembly process.

We prepared colloidal monolayers with polystyrene (PS) microspheres of $3 \mu \mathrm{m}$ and $840 \mathrm{~nm}$ diameter, respectively. The typical size of the monolayers was in the order of several $\mathrm{mm}^{2}$ up to $1 \mathrm{~cm}^{2}$. From those monolayers stamps were casted and used for $\mu$-CP. After thiols were transferred to a gold substrate we obtained hexagonal surface domains as confirmed by lateral force microscopy. The bright and dark areas in Fig. 1 correspond to hydrophilic and hydrophobic regions, respectively.

\section{Experimental results}

When the sol is applied by dip-coating to a gold surface patterned with a stamp made from spheres of $3 \mu \mathrm{m}$ in diameter, a periodic structure is formed as suggested already by the occurrence of interference colors which are visible with the bare eye and confirmed with an optical microscope. Fig. 2 shows an atomic force microscope image of the sample after it was annealed at $120^{\circ} \mathrm{C}$ for about $30 \mathrm{~min}$. Obviously, the sol dewetted completely the hydrophobic sites (see also Fig. 1), thus producing monodisperse, hexagonally arranged drops where during the subsequent annealing step a chemical reaction, i.e. the sol-gel transition, takes place. As a result, one obtains $\mathrm{WO}_{3}$ dots which strongly adhere to the surface and withstand an ultrasonic bath treatment of $1 \mathrm{M}$ hydrochloric acid. In order to demonstrate that these droplets exhibit chromogenic properties we first evaporated a thin layer of palladium and then exposed the structure to hydrogen gas. We observed an immediate

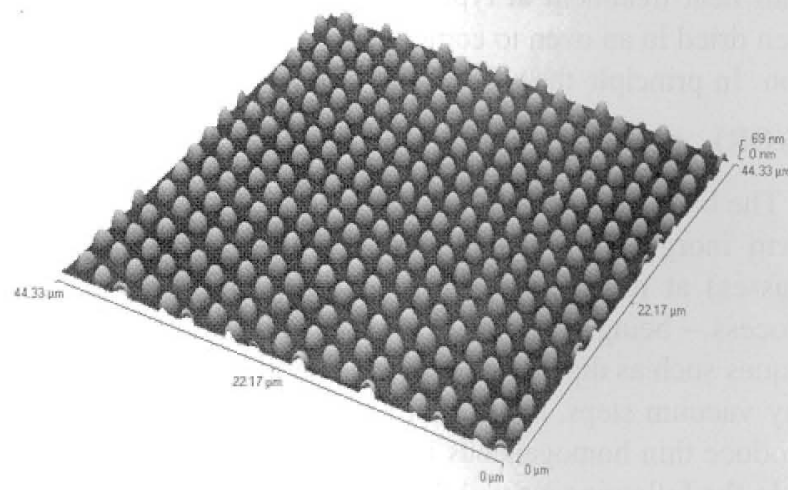

Fig. 2. Atomic force microscopy (AFM) image of an array of tungsten oxide dots obtained by dip coating of tungsten alkoxide to a chemically patterned surface. After an annealing step at $120^{\circ} \mathrm{C}$, the liquid drops are transformed into solid dots. 


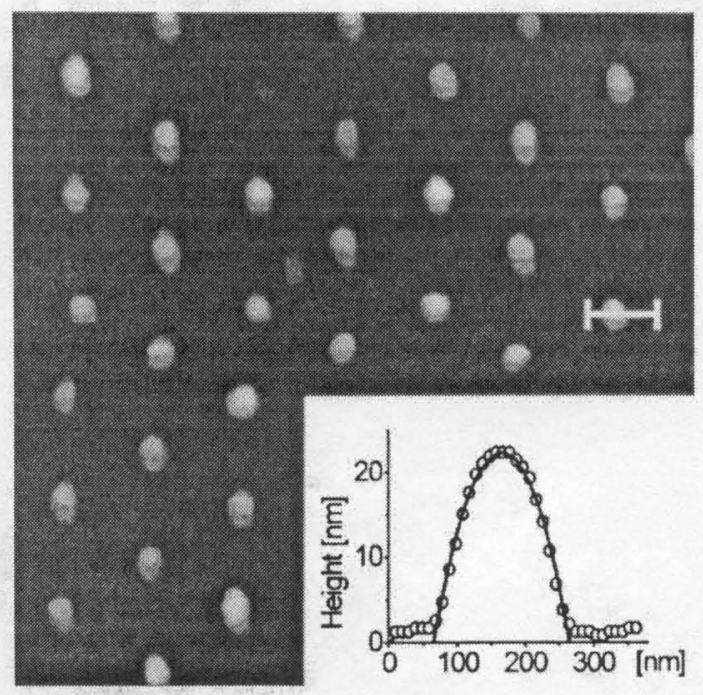

Fig. 3. AFM image $\left(3.73 \times 3.75 \mu \mathrm{m}^{2}\right)$ of tungsten oxide dots fabricated by dip coating on a gold surface prepatterned with a stamp made from a monolayer of $600 \mathrm{~nm}$ microspheres. The inset shows a cross section of a dot after the annealing process which can be fitted to a spherical cap (solid line). The typical size (FWHM) of the dots is about $150 \mathrm{~nm}$.

change in the reflected intensity which is typical for tungsten oxide thin films [25].

An array of $\mathrm{WO}_{3}$ dots formed on a substrate prepatternd with a stamp molded from a monolayer of $600 \mathrm{~nm}$ PS spheres is shown in Fig. 3. After the annealing process, the typical full width half maximum (FWHM) of the dots is in the order of $150 \mathrm{~nm}$ as can be seen from a cross section in the inset. The profile of the solid dots fits very well to a spherical cap function (solid line) which is typically obtained for liquid drops. Obviously the polycondensation does not alter the shape of the dot which is in agreement with the fact that tungsten oxide forms an amorphous network at these temperatures $[20,26]$. Only above $350^{\circ} \mathrm{C}$ where crystallization occurs, deviations from a spherical shape are observed.

Fig. 4 shows an example where sol was allowed to rest on

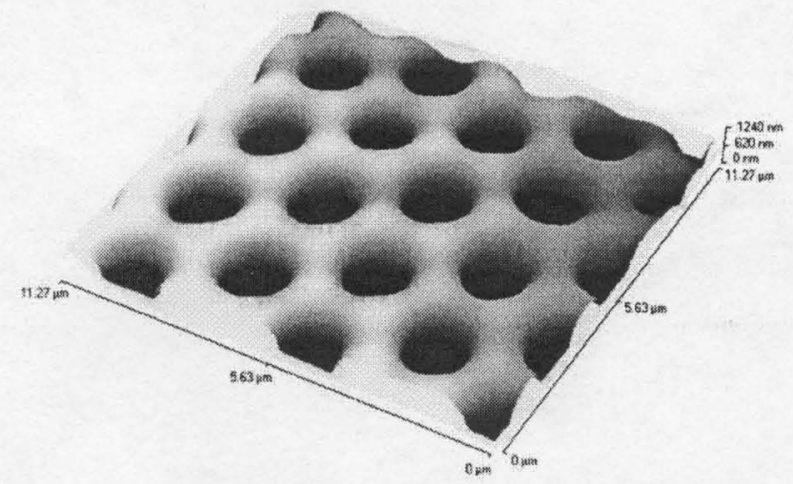

Fig. 4. Inverse structure, which is obtained when the sol is not applied by dip coating but is allowed to rest on the chemically patterned substrate. The substrate prepattern was obtained by a colloidal monolayer of polystyrene spheres of $3 \mu \mathrm{m}$ diameter. the substrate for about $15 \mathrm{~s}$ before it was removed by tilting the substrate. Although exactly the same recipe for the sol was used, now the 'inverse' structure of Fig. 2 is obtained. While the details of this process are not understood in detail yet, we believe that the formation of this geometry is due to micro-phase separation between the surfactant and the sol. When the sol rests at the substrate, the acetylacetone might preferentially wet the hydrophilic sites, thus leaving only the hydrophobic areas for the tungsten alkoxide. Thus, just by a the details how the sol is applied to the surface a completely different surface structure may be obtained.

\section{Conclusions}

In summary we have shown that the dewetting of tungsten alcoholates on surfaces which are prepatterned via microcontact printing leads to the formation of small drops. Due to the high reactivity of this material a subsequent chemical reaction, i.e. a sol-gel process, occurs and finally leads to the formation of solid tungsten oxide dots. We also demonstrated that by simply changing the process how the alkoxide is applied to the surface either the 'positive' or the 'inverse' structure is obtained. The results presented here should also apply to many other materials which can be produced by the sol-gel technique. Since the approach presented here does not rely on vacuum techniques it might be a convenient way of nanostructure fabrication.

\section{Acknowledgements}

This work was supported by the Deutsche Forschungsgemeinschaft, SFB 513.

\section{References}

[1] C.J. Brinker, G.W. Scherer, Sol-gel Science, Academic Press, San Diego, CA, 1990.

[2] S. Katayama, M. Sekine, H. Fudouzi, M. Kuwabara, J. Appl. Phys. 71 (1992) 2795.

[3] C. Sanchez, J. Livage, M. Henry, F. Babonneau, J. Non-Cryst. Sol. 100 (1988) 65.

[4] C.J. Brinker, A.J. Hurd, G.C. Frye, K.J. Ward, C.S. Ashley, J. NonCryst. Sol. 121 (1990) 294.

[5] Y. Xia, J. Tien, D. Qin, G.M. Whitesides, Langmuir 12 (1996) 4033.

[6] G.P. Lopez, H.A. Biebuyck, C.D. Frisbie, G.M. Whitesides, Science 260 (1993) 647.

[7] A. Kumar, G. Whitesides, Science 263 (1994) 60.

[8] Z. Huang, P.-C. Wang, A.G. MacDiarmuid, Y. Xia, G. Whitesides, Langmuir 13 (1997) 6480.

[9] M. Böltau, S. Walheim, J. Mlynek, G. Krausch, U. Steiner, Nature 391 (1998) 877.

[10] H. Yang, N. Coombs, G.A. Ozin, Adv. Mater. 9 (1997) 811.

[11] C. Marzolin, S.P. Smith, M. Prentiss, G.M. Whitesides, Adv. Mater. 10 (1998) 571.

[12] P. Yang, T. Deng, D. Zhao, et al., Science 282 (1998) 2244.

[13] S.K. Deb, Philos. Mag. 27 (1973) 801.

[14] R.S. Crandall, B.W. Faughnan, Phys. Rev. Lett. 39 (1977) 232. 
[15] C. Bechinger. S. Herminghaus, P. Leiderer, Thin Solid Films 239 (1994) 156.

(16) A.I. Gavrilyuk, T.G. Lanskaya, F.A. Chudnovskii. Sov. Phys. Tech. Phys. 32 (1987) 964.

[17] C.G. Granqvist, Sol. State Mater. Sci. 16 (1990) 291.

[18] C. Bechinger. S. Ferrere, A. Zaban, J. Sprague, B.A. Gregg, Naiure 383 (1996) 608 .

[19] M. Gugliclmi. G. Carturan, J. Non-Cryst. Sol. 100 (1988) 16

[20] P. Judenstein, R. Morineau. J. Livage, Solid State lonics 51. (1992) 239.
[21] M.A. Hábib, D. Glueck, Sol. Energy Mater. 18 (1989) 127.

[22] N.D. Denkov. O.D. Velev, P.A. Kralchevsky, I.B. Ivanor, H. Yoshimura, K. Nagayama, Langmuir 8 (1992) 3183 .

[23] U.C. Fischer, H.P. Zingsheim, J. Vac. Sci. Technol. 19 (1981) 881.

[24] F. Bunneister, C. Schäfle, B. Keilhofer, C. Bechinger, J. Boneberg, P. Leiderer, Adv. Mater. 10 (1998) 495.

[25] K. Ito, T. Ongarni, Appl. Phys. Lett. 60 (1992) 938.

[26] M. Shiojiri, T. Miyano, C. Kailo. Jpn. J. Appl. Phys. 17 (1978) 567. 